\title{
Five dimensional perfect fluid coupled with massless scalar field cosmological model in GTR
}

\author{
Kalpana pawar (Mody) ${ }^{\mathrm{a}}$, Vidhya Chauhan ${ }^{\mathrm{b}}$, G. D. Rathod ${ }^{\mathrm{c}}$, R. V. Saraykar ${ }^{\mathrm{d}}$ \\ ${ }^{I}$ (Department of Mathematics, Shivaji Science College, Nagpur, India) \\ ${ }^{2}$ Department of Mathematics RTM Nagpur University, Nagpur, India.)
}

\begin{abstract}
The field equations for perfect fluid coupled with mass less scalar field are solved with conditions $p=\rho$ and $R=k A^{n}$ (where $k$ and $n$ both are constant.) for five dimensional space-time in General Theory of Relativity. Also various physical and geometrical properties of the model have been discussed.
\end{abstract}

Keywords - Five dimensional space-time, mass less scalar field, perfect fluid

\section{INTRODUCTION}

Any physical theory can be studied easily through the exact solutions of its mathematical structure. Therefore, the exact solutions of relativistic model plays an important role than those obtained through approximations scheme and numerical computations. Moreover, the use of various symmetries leads to physical viable information from the complicated structure of the field equations in Einstein's theory. The gravitational effect of cylindrically symmetric interacting mass less scalar field is a subject of current interest because of it's possible applications to nuclear physics. The causes of coupled sources free electromagnetic fields and stiff fluid distributions are equivalent to mass less scalar fields was investigated by Mohanty, Tiwari and Rao[8]. The origin of structure in the universe is one of the greatest cosmological mysteries even today. The existence of a large scale network of strings in the early universe is not a contradiction with the present day observations of the universe. The present day observations indicate that the universe at large scale is homogeneous and isotropic and it is accelerating phase of the universe (recently detected experimentally by Gasperni [1]. It is well known that exact solutions of general theory of relativity for homogeneous space-time belong to either Bianchi type or Kantowski-Sachs by Roy and Choudhary[17]. Weber [20,21] had done a qualitative study of Kantowski-Sachs. Lorenz [5,6] has obtained exact Kantowski-Sachs vacuum models in Brans-Dicke theory, while Sing and Agrawal discussed Kantowski-Sachs type models in Seaz and Ballester. In particular Reddy discussed some string models in Seaz and Ballester scalar- tensor theory of gravitation in four dimensions.

Recently, Reddy[16,17] presented a string cosmological model in Brans-Dicke and Seaz-Ballester Scalar-tensor theories of gravitation. Also the mass less scalar field in relativistic mechanics yields some significant results regarding both the singularities involved and Mach's principle. Panigrahi and Sahu [11] studied a micro and macro cosmological model in the presence of mass less scalar field interacted with the perfect fluid.

In this paper, we have studied cosmological model generated by perfect fluid coupled with mass less scalar field for five dimensional space-time in general theory of relativity. For solving field equations, the relation $p=\rho$ and relation between metric potentias $\mathrm{R}=\mathrm{kA}^{\mathrm{n}}$ are used. Some physical and geometrical properties of the determinate model are also discussed.

\section{The Field Equations}

We consider the five dimensional line element in the form

$d s^{2}=R^{2}\left(d X^{2}+d Y^{2}+d Z^{2}\right)+A^{2} d \psi^{2}-d T^{2}$

Where $R$ and $A$ are functions of cosmic time $t$ only.

The relativistic field equations for coupled perfect fluid and mass less scalar fields are,

$G_{i j}=R_{i j}-\frac{1}{2} g_{i j} R=-\left(T_{i j}+U_{i j}\right)$

where $T_{i j}$ is the stress energy tensor corresponding to perfect fluid and $U_{i j}$ is the stress energy tensor corresponding to massless scalar field respectively.

$T_{i j}=(\rho+p) u_{i} u_{j}-p g_{i j}$ and

$U_{i j}=V_{; i} V_{, i}-\frac{1}{2} g_{i j} V_{, k} V^{k}$,

where $g_{i j} u_{i} u^{j}=1$, and 
$g^{i j} V_{; i j}=0$.

Here $u^{i}$ is the four velocity vector of the fluid, $p$ and $\rho$ are the proper pressure and energy density respectively. Comma (,) and semicolon (;) denotes partial and covariant differentiation respectively.

From equations (3) and (4) the non-vanishing components of $T_{i j}$ and $U_{i j}$ are

$T_{0}^{0}=T_{1}^{1}=T_{2}^{2}=T_{3}^{3}=-p, \quad T_{4}^{4}=\rho \quad$ and

$U_{0}^{0}=U_{1}^{1}=U_{2}^{2}=U_{3}^{3}=\frac{1}{2} V_{4}^{2}, \quad U_{4}^{4}=-\frac{1}{2} V_{4}^{2}$

With the help of equations (7) and (8), the relativistic field equations (2), can be written as,

$\frac{2 R_{44}}{R}+\frac{R_{4}^{2}}{R^{2}}+\frac{2 R_{4} A_{4}}{R A}+\frac{A_{44}}{A}=p-\frac{1}{2} V_{4}^{2}$,

$\frac{3 R_{44}}{R}+\frac{3 R_{4}^{2}}{R^{2}}=p-\frac{1}{2} V_{4}^{2}$ and

$\frac{3 R_{4}^{2}}{R^{2}}+\frac{3 R_{4} A_{4}}{R A}+\frac{A_{44}}{A}=-\rho+\frac{1}{2} V_{4}^{2}$.

The suffix ' 4 ' denotes ordinary differentiation with respect to time ' $t$ '.

\section{Solutions of Field Equations and Physical Properties}

The set of equations $(9-11)$ contains three equations with five unknowns $R, A, \rho, p$ and $V$. For complete determinacy of the system, two extra conditions are needed. We assume equation of state in the form,

$p=\rho, \quad$ and

a relation between metric coefficient (potentials) $R$ and $A$ as,

$R=k A^{n}$,

where ' $A$ ' and ' $n$ ' are both constants.

Adding equations (9) and (11), and using equation (12), we get

$\frac{2 R_{44}}{R}+\frac{4 R_{4}^{2}}{R^{2}}+\frac{5 R_{4} A_{4}}{R A}+\frac{A_{44}}{A}=0$.

Adding equations (10) and (11), and using equation (12), we get

$\frac{2 R_{44}}{R}+\frac{4 R_{4}^{2}}{R^{2}}+\frac{2 R_{4} A_{4}}{R A}=0$.

Subtracting equation (14) from equation (15), we get

$\frac{3 R_{4} A_{4}}{R A}+\frac{A_{44}}{A}=0$.

Using equation (13), in equation (16), we get

$3 n\left(\frac{A_{4}}{A}\right)^{2}+\frac{A_{44}}{A}=0$.

The solution of equation (17), is given by

$A=N\left(k_{1} t+k_{2}\right)^{\frac{1}{3 n+1}}$

where $N=(3 n+1)^{\frac{1}{3 n+1}}$.

Using equation (18), equation (13) becomes

$R=M\left(k_{1} t+k_{2}\right)^{\frac{n}{3 n+1}}$,

where $M=k N^{n}$

From equation (17), we get

$V_{44}+V_{4}\left[\frac{3 R_{4}}{R}+\frac{A_{4}}{A}\right]=0$.

Using (18) and (19), in equation (20), we get

$\frac{V_{44}}{V_{4}}+\frac{k_{1}}{k_{1} t+k_{2}}=0$.

$\frac{V_{44}}{V_{4}}+\frac{k_{1}}{T}=0$

where $T=k_{1} t+k_{2}$

On integrating equation (22), we get

$V_{4} T=k_{3}$,

where, $k_{3}$ is constant of integration.

Solving equation (23), we get

$V=k_{4} \log T+k_{5}$

where $k_{4}=\frac{k_{3}}{k_{1}}$. 
Here $k_{4}$ and $k_{3}$ are constants of integration.

Using equations (18) and (19), the line element (1) becomes

$$
\begin{array}{r}
d s^{2}=M^{2}\left(k_{1} t+k_{2}\right)^{\frac{2 n}{3 n+1}}\left(d X^{2}+d Y^{2}+d Z^{2}\right) \\
+N^{2}\left(k_{1} t+k_{2}\right)^{\frac{2}{3 n+1}} d \psi^{2}-d T^{2} .
\end{array}
$$

This metric can be transformed through a proper choice of coordinates to the form

$d s^{2}=M^{2} \frac{2 n}{3 n+1}\left(d X^{2}+d Y^{2}+d Z^{2}\right)+N^{2} \frac{2}{3 n+1} d \psi^{2}$

$$
-d T^{2} \text {. }
$$

where $k_{1} t+k_{2}=T$.

\section{Some Physical and Geometrical Properties}

The pressure $p$ and density $\rho$ in the model (26) are given by

$p=\rho=\frac{k_{3}^{2}}{2 T^{2}}-\frac{3 n(n+1)}{(3 n+1)^{2}} \frac{k_{1}^{2}}{T^{2}}$,

Spatial volume $v^{3}=\sqrt{-g}=N M^{3} T$,

Scalar expansion $\theta=\frac{1}{T}$,

Shear expansion $\sigma^{2}=\frac{2}{9 T^{2}}$ and

Deceleration parameter $q=2>0$.

The model (26) has no initial singularity, while the energy density $\rho$, pressure $p$ given by (27) possess initial singularities. However, as $T$ increases these singularities vanish. The spatial volume of the model given by (28) shows anisotropic expansion of the universe (26) with time. For model (26), the expansion scalar $\theta$ and shear scalar $\sigma$ tends to zero as $T \rightarrow \infty$. The positive value of the deceleration parameter indicates that the model decelerates in standard way.

Also, since $\lim _{T \rightarrow \infty} \frac{\sigma}{\theta} \neq 0$.

The model does not approach an isotropy for large values of $T$.

\section{Conclusion}

We have studied cosmological models generated by perfect fluid coupled with mass less scalar field for five dimensional space-time in General Theory of Relativity. The model is free from singularities and it is expanding, anisotropic and decelerates in the standard way. Also we find that all the physical quantities like pressure and density diverges at the initial moment of creation.

1] Gasperini, M.: Phys. Rep. 373, 1(2003)

\section{References}

[2] Gron, P.: Math. Phys 27, 1490 (1986)

[3] Krori, K. D., Goswami, A. K., Purkayastha, A. D.: J. Math. Phys. 36, 1347 (1995)

[4] Li, X. Z., Hao, J. G.: Phys. Rev. D. 68, 083512 (2003)

[5] Lorenz, D.: Astrophys.Space Sci.98, 101(1984)

[6] Lorenz, D.: J. Phys. A: Math. Gen. 16, 575 (1983)

[7] Matravers, D. R.: Gen. Relativ. Gravit. 20, 279 (1988)

[8] Mohanty, G., Tiwari, R. N. Rao, J. R.: Int.J. Theor. Phys. 21, 105 (1982)

[9] Mohanty. G., Mahanta K.L. and .Bishi B.K.,Astrophys Space Sci., 310(2007)273.

[10] Mohanty. G.,Mahanta K.L. and Sahoo R.R.,Astrophys.Space Sci., 306(2006)269

[11] Panigrahi, U. K., Sahu, R. C.: Theor. Appl. Mech. 30(3) , 163-175(2003)

[12] Rahaman F., Bag G.,Bhui B.C., and Das S., Fizika B12, (2003b)193

[13] Rahaman, F., Chakraborty S., Das S., Hossian M. And Bera J., Pramana J. Phys. 60(2003a)453

[14] Rahaman, F.,Chakraborty S.and Bera J.,Int.J. Mod. Phys. D11(2002)1501

[15] Reddy, D. R. K.: Astrophys. Space Sci. 286, 365 (2003)

[16] Reddy, D. R. K.: Astrophys. Space Sci. 286, 397 (2003)

[17] Roy, Choudhary, A.K.: Theoretical Cosmology. Claredon,Oxford (1979)

[18] Seaz, Ballester, V. J.: Phys. Lett. A 113, 467 (1985)

[19] Walecka, J. D.:Ann. Phys. 83, 491 (1974)

[20] Weber, E.: J. Math. Phys. 25, 3279 (1984)

[21] Weber, E.: J. Math. Phys. 26, 1308 (1985) 